http://dx.doi.org/10.35381/racji.v5i3.1104

\title{
Preservación de la evidencia digital en el procedimiento de Cadena de Custodia
}

\section{Preservation of digital evidence in the Chain of Custody procedure}

\author{
Franklin Washington Montecé-Mosquera \\ db.franklinwmm42@uniandes.edu.ec \\ Universidad Regional Autónoma de los Andes, Babahoyo \\ Ecuador \\ https://orcid.org/0000-0002-8905-3197 \\ José Augusto Izquierdo-Montecé \\ db.joseaim17@uniandes.edu.ec \\ Universidad Regional Autónoma de los Andes, Babahoyo \\ Ecuador
}

Recibido: 31 de octubre de 2020

Revisado: 10 de octubre de 2020

Aprobado: 05 de diciembre de 2020

Publicado: 10 de diciembre de 2020 


\title{
RESUMEN
}

La presente investigación tiene como objetivo identificar las dificultades que se presentan en el procedimiento de cadena de custodia de la evidencia digital, que, en algunos casos, provocan la contaminación de la prueba. El estudio desde la perspectiva cuantitativa, de tipo descriptiva se complementa por un diseño documental-bibliográfico, que parte de criterios particulares de los fenómenos relacionados con la cadena de custodia que, con los resultados obtenidos, se propende a establecer cambios que permitan mejorar los procedimientos de control y manejo adecuado de la evidencia digital. Se trabajó con una población de 94 personas, relacionas al trabajo en la Fiscalía de los Ríos, que conforman la muestra, a los cuales se les aplicó un cuestionario. Como resultado del trabajo realizado nos permite llegar a la conclusión de que el personal especializado de la Policía Nacional no tiene conocimientos actualizados en el uso de la tecnológica para custodiar las evidencias digitales.

Descriptores: Procedimiento legal; crimen; sanción penal. (Palabras tomadas del Tesauro UNESCO).

\begin{abstract}
The objective of this research is to identify the difficulties that arise in the chain of custody procedure for digital evidence, which, in some cases, cause contamination of the evidence. The study from the quantitative perspective, of a descriptive type, is complemented by a documentary-bibliographic design, which starts from particular criteria of the phenomena related to the chain of custody that, with the results obtained, tends to establish changes that allow improving the procedures control and proper management of digital evidence. We worked with a population of 94 people, related to work in the Prosecutor's Office of the Rivers, that make up the sample, to whom a questionnaire was applied. As a result of the work carried out, we can conclude that the specialized personnel of the National Police do not have up-to-date knowledge in the use of technology to guard digital evidence.
\end{abstract}

Descriptors: Legal procedure; crime; criminal sanction. (Words taken from the UNESCO Thesaurus). 
lustitia Socialis. Revista Arbitrada de Ciencias Jurídicas.

Año V. Vol. V. №3. Edición Especial. 2020-III:

Universidad Regional Autónoma de los Andes

Hecho el depósito de Ley: FA2016000064

ISSN: 2542-3371

FUNDACIÓN KOINONIA (F.K). Santa Ana de Coro, Venezuela

Franklin Washington Montecé-Mosquera; José Augusto Izquierdo-Montecé

\section{INTRODUCCIÓN}

Para sustentar la efectividad de una acusación ante la Fiscalía, es fundamental la aplicación correcta de la cadena de custodia dentro de la investigación procesal, considerando que aquellos indicios y/o evidencias digitales serán establecidas como prueba para que el Fiscal la refiera dentro del proceso que después de su presentación, puedan influir en el criterio del magistrado a cargo del proceso. En este sentido, la informática, ha conquistado la mayoría de las sociedades humanas, sin distinciones políticas, sociales, ni religiosas. Para Calderón-Arias (2014), en Latinoamérica los estados por medio de sus constituciones establecen los mecanismos necesarios para este fin:

Resulta común en Latinoamérica que las leyes adjetivas penales de las respectivas naciones siquiera conceptualicen la cadena de custodia de los elementos de prueba, solamente se limitan a recoger ciertas actividades, fundamentalmente relacionadas con los medios de prueba y los peritos, recogen las características de la cadena de custodia, pues es innegable la necesidad de la presencia de ésta en pos de la conservación del material probatorio que se obtenga. (p.249)

Se evidencia que en los últimos años la tecnología ha evidenciado un incremento progresivo en el uso de equipos informáticos y el acceso a las redes de telecomunicaciones con acceso a internet, esto ha causado el significativo incremento de problemas conocidos como incidentes de seguridad informática (Sampaoli, 2018). Llegando a introducirse efectivamente en millones de hogares en las más diversas latitudes y longitudes, amenazando con dividir al mundo en dos grandes grupos principales; los que tienen acceso a la información y los que carecen de ella. Ha surgido un nuevo tipo de pobres que difícilmente puedan superar esta dificultad.

En materia constitucional, el articulo 76 en relación a lo establecido a las garantías al debido proceso, incida: "Las pruebas obtenidas o actuadas con violación de la Constitución o la ley no tendrán validez alguna y carecerán de eficacia probatoria”, es importante que el procedimiento para aplicar una correcta cadena de custodia en dispositivos informáticos o digitales sea claro y preciso, todo esto, para lograr que se asegure al máximo la conservación de los indicios y evidencias obtenidas durante la 
ejecución de la acción, el cual, aunque en la actualidad se aplica en ciertos casos, todavía presenta falencias en lo que corresponde al reclutamiento del personal capacitado para cumplir eficientemente su trabajo en esa área (Constitución de la República del Ecuador, 2008). Por ello, la cadena de custodia se considera con reiteración como un procedimiento de control.

\section{Antecedentes relacionados con la investigación}

Considerando que los antecedentes, son aquellas investigaciones realizadas con anterioridad o que preceden al trabajo que se está desarrollando y que además guardan similitud con el objetivo propio de la investigación, se consideran los siguientes trabajos realizados:

El autor Acurio Del Pino (2015) establece en el Manual elaborado que: la obtención de Información considerada como elementos de convicción, se constituye en una de las facetas útiles dentro del éxito de en una investigación criminal, con la finalidad de encaminar correctamente la investigación, se determina la obtención de indicios y posteriormente los elementos probatorios suficientes para fundamentar la acusación formal.

Es este mismo orden de ideas, Escobar-De León (2017) indica en su trabajo Manejo de la Cadena de Custodia en la Recolección de Evidencia Digital indica:

La evidencia digital reúne requisitos similares a la evidencia física, aunque su forma y tangibilidad varíen de forma puntual. La relación causal entre la innovación tecnológica y su influencia en la vida del ser humano denotan las circunstancias de la utilidad de las herramientas o dispositivos digitales para cualquier actividad que se realice en la actualidad. Desde redactar un documento digital con fines personales, hasta el desarrollo de software avanzado para industrializar procesos de trabajo o de producción, esto enmarca el ámbito de aplicación de la tecnología y sus dispositivos en la vida del ser humano en el presente. Por lo que es indispensable que al abordar el contenido de la evidencia digital se contenga el potencial de la mente abierta para delimitar su utilidad e importancia en la investigación criminal. 
La evidencia digital reúne requisitos similares a la evidencia física, aunque su forma y tangibilidad varíen de forma puntual. La relación causal entre la innovación tecnológica y su influencia en la vida del ser humano denotan las circunstancias de la utilidad de las herramientas o dispositivos digitales para cualquier actividad que se realice en la actualidad. Desde redactar un documento digital con fines personales, hasta el desarrollo de software avanzado para industrializar procesos de trabajo o de producción, esto enmarca el ámbito de aplicación de la tecnología y sus dispositivos en la vida del ser humano en el presente. Por lo que es indispensable que al abordar el contenido de la evidencia digital se contenga el potencial de la mente abierta para delimitar su utilidad e importancia en la investigación criminal.

Posterior a la recopilación de las evidencias digitales aún se corre riesgo de que éstas pierdan su validez, ya que no se aplica un procedimiento adecuado de control recurrente de todas las muestras de evidencia recolectadas, mismas que, por no estar a buen recaudo en depósitos idóneos de preservación que en la actualidad están bajo responsabilidad de la Policía Judicial para su custodia sin aplicar los protocolos sugeridos; pueden perder su eficacia por el deterioro, lo que derivaría en el aplazamiento o, dependiendo del caso, el juicio caería en un ralentizamiento innecesario que no permitiría lograr un fallo verídico y oportuno. Todas esas situaciones provocan que la evidencia digital se contamine y eso impida determinar la culpabilidad o inocencia del acusado debido al inadecuado protocolo aplicado en la cadena de custodia de la evidencia digital.

En tal sentido, la ausencia de un trabajo claro y preciso sobre el procedimiento que implica implementar la cadena de custodia en la investigación de delitos informáticos, para asistir a la detección de las deficiencias en la preservación de la evidencia digital, como elemento fundamental dentro de los procesos judiciales. En apoyo de otras ciencias y el uso de la tecnología constituyen un gran apoyo para procesar las evidencias en varios campos de las ciencias forenses, al respecto, los autores (Serrano-Hernández, Serrano-Gómez \& Serrano-Gómez, 2017), indican:

Las características de la actividad médico legal, en especial de aquellas que van dirigida a los procesos de control y manejo de datos de la información, han generado una nueva perspectiva del fenómeno que 
llevan a considerar cómo hacer para tratar todo el volumen de información que emana de cada caso asistido desde el Departamento de Medicina Legal para dar respuesta a la diversidad de informes estadísticos (p. 823)

\section{Actualidad e importancia}

En términos generales, la cadena de custodia emplea normas de procedimiento que son utilizadas por los responsables de la custodia de la evidencia física, garantizan tres elementos fundamentales: La preservación, confiabilidad y registro del responsable, considerando que es su deber custodiar las evidencias de una manera profesional y responsable (Coello, 2017).

A pesar del avance tecnológico, en la actualidad en algunas instituciones del sistema judicial los procesos de preservación y custodia de la evidencia digital se realizan con prácticas rústicas, que no cumplen o no cuentan con las normas adecuadas o poco regularizadas con estándares.

Se considera a la cadena de custodia como un conjunto de procedimientos susceptibles a errores, poniendo en juego la importancia de la participación del personal destinado para la custodia y preservación de la evidencia digital, ya que con esta base de elementos la Fiscalía General del Estado, por medio de su representante, podrá determinar con elementos suficientes de convicción la materialidad de la infracción y establecer responsabilidades de los involucrados en el cometimiento del delito enmarcado en las características determinadas en el crimen informático.

En el Ecuador es necesario un procedimiento de análisis adecuado para la preservación de la evidencia digital, considerando que no se utilizan los medios efectivos para que se genere el efectivo cuidado que hace que la evidencia digital se contamine, lo que genera insuficiencias en la etapa de investigación para posteriormente determinar correctos indicios de responsabilidad.

El objetivo del presente trabajo de investigación es identificar las dificultades que se presentan en el procedimiento de cadena de custodia de la evidencia digital. 


\section{METODOLOGÍA}

El estudio desde la perspectiva cuantitativa, de tipo descriptiva se complementa por un diseño documental-bibliográfico, que parte de criterios particulares de los fenómenos relacionados con la cadena de custodia que, con los resultados obtenidos, se propende a establecer cambios que permitan mejorar los procedimientos de control y manejo adecuado de la evidencia digital. (Parela-Stracuzzi \& Martins-Pestana, 2012). permitirá analizar la forma de cómo se maneja la cadena de custodia de la evidencia digital en Fiscalía Los Ríos - Babahoyo, facilitando la identificación de las falencias que posee actualmente, así mismo permitió la recogida de información extraída de los diferentes textos jurídicos abordando el método analítico enfocándose en el estudio de aspectos particulares, examinados en el análisis de contenido escrito, mediante lo analítico-sintético (Bernal-Torres, 2006), obteniendo nuevos conocimientos de la descomposición mental en varios elementos. Se utilizó una población de 94 personas, que conforman la muestra, distribuidos de la siguiente manera: Agentes Fiscales de la Fiscalía Provincial de Los Ríos 40, Secretarios de Fiscalía 40, Peritos calificados 6, Policía especializada (PJ) 8; a los cuales se les aplicó un cuestionario, y se obtuvieron datos que nos dio como resultado el porcentaje de las personas que conocen y cuantas desconocen la cadena de custodia y la injerencia en el cuerpo legal.

\section{RESULTADOS}

A continuación, se presentan los resultados obtenidos:

1. ¿Cree usted que el personal especializado de la Policía Nacional tiene conocimientos actualizados en el uso de la tecnológica para custodiar las evidencias digitales? 


\section{Tabla 1.}

\begin{tabular}{|l|c|c|}
\hline OPCIONES & NÚMERO & PORCENTAJE \\
\hline SI & 10 & $10 \%$ \\
\hline NO & 86 & $90 \%$ \\
\hline TOTAL & 96 & $100 \%$ \\
\hline
\end{tabular}

Fuente: Resultado de la encuesta. Elaboración Propia (2020).

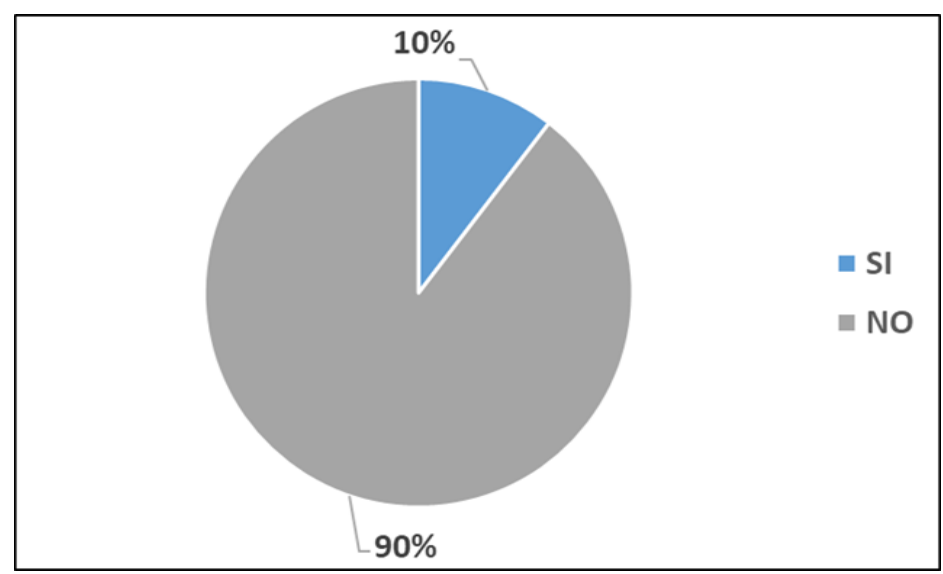

\section{Gráfica 1.}

Fuente: Resultado de la encuesta. Elaboración Propia (2020).

El resultado de las respuestas obtenidas nos permite llegar a la conclusión de que el personal especializado de la Policía Nacional no tiene conocimientos actualizados en el uso de la tecnológica para custodiar las evidencias digitales. 
2. ¿Considera que la manipulación sin protocolos de seguridad de los indicios identificados, deriva en la modificación de la prueba?

Tabla 2.

\begin{tabular}{|l|c|c|}
\hline OPCIONES & NÚMERO & PORCENTAJE \\
\hline SI & 90 & $94 \%$ \\
\hline NO & 6 & $6 \%$ \\
\hline TOTAL & 96 & $100 \%$ \\
\hline
\end{tabular}

Fuente: Resultado de la encuesta. Elaboración Propia (2020).

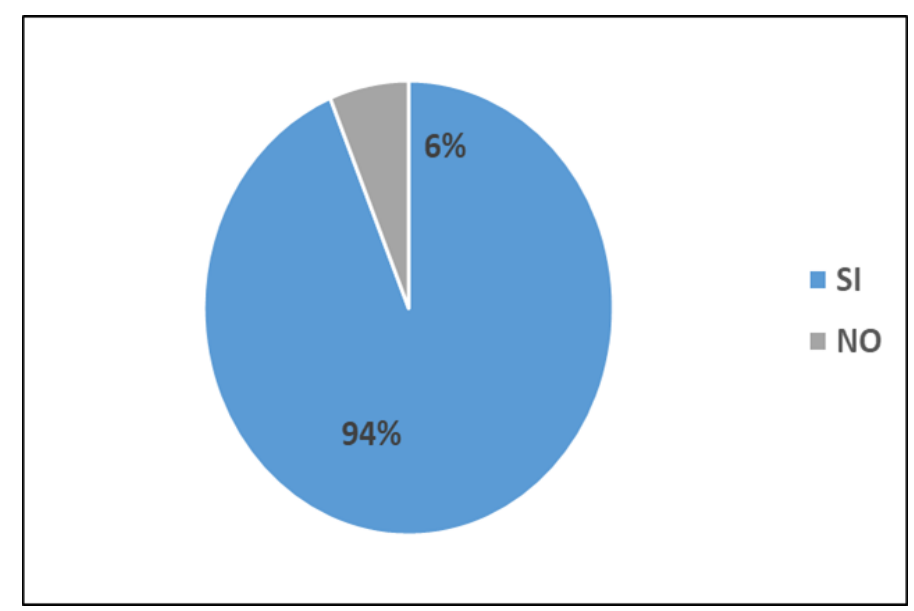

\section{Gráfico 2.}

Fuente: Resultado de la encuesta. Elaboración Propia (2020).

Se evidencia que la apreciación mayoritaria de los encuestados es que la manipulación sin protocolos de seguridad de los indicios identificados, deriva en la modificación de la prueba provocando una inminente contaminación de la misma. 
3. ¿Con qué frecuencia recibe capacitación sobre los nuevos procedimientos de custodia de evidencia digital?

Tabla 3.

\begin{tabular}{|c|c|c|}
\hline OPCIONES & NÚMERO & PORCENTAJE \\
\hline ALTO & 0 & $0 \%$ \\
\hline MEDIO & 15 & $16 \%$ \\
\hline BAJO & 35 & $36 \%$ \\
\hline NINGUNO & 46 & $48 \%$ \\
\hline TOTAL & 96 & $100 \%$ \\
\hline
\end{tabular}

Fuente: Resultado de la encuesta. Elaboración Propia (2020).

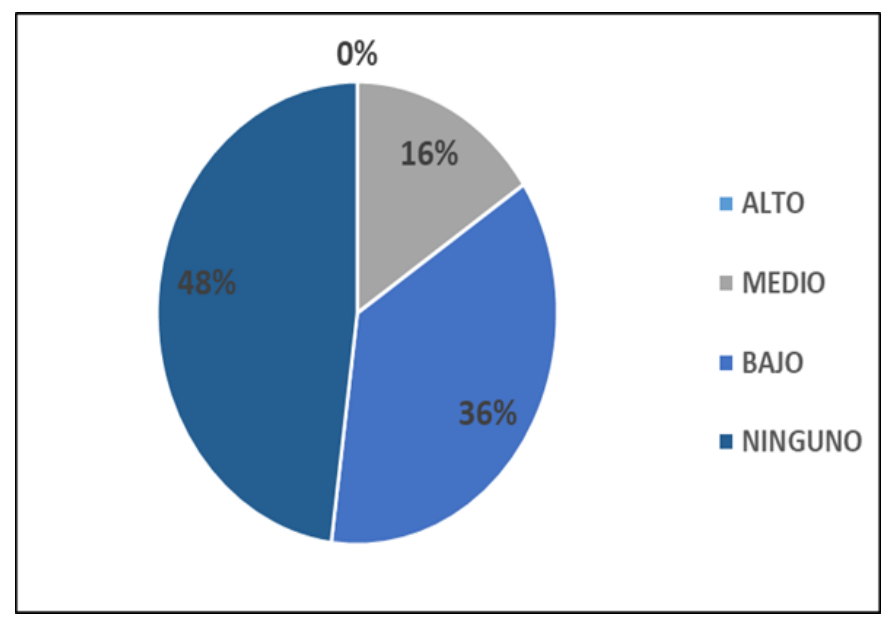

Gráfica 3.

Fuente: Resultado de la encuesta. Elaboración Propia (2020).

Un alto número del grupo encuestado está consciente de que la capacitación sobre los nuevos procedimientos de custodia de evidencia digital es una necesidad urgente, en el cantón Babahoyo para lograr un efectivo control y aplicación de los procedimientos en la preservación de la evidencia digital. 
4. ¿Considera que el juez podrá aplicar la sana crítica para emitir la sentencia si se comprueba que la evidencia digital ha sido manipulada?

Tabla 4.

\begin{tabular}{|l|c|c|}
\hline OPCIONES & NÚMERO & PORCENTAJE \\
\hline SI & 0 & $0 \%$ \\
\hline NO & 96 & $100 \%$ \\
\hline TOTAL & 96 & $100 \%$ \\
\hline
\end{tabular}

Fuente: Resultado de la encuesta. Elaboración Propia (2020).

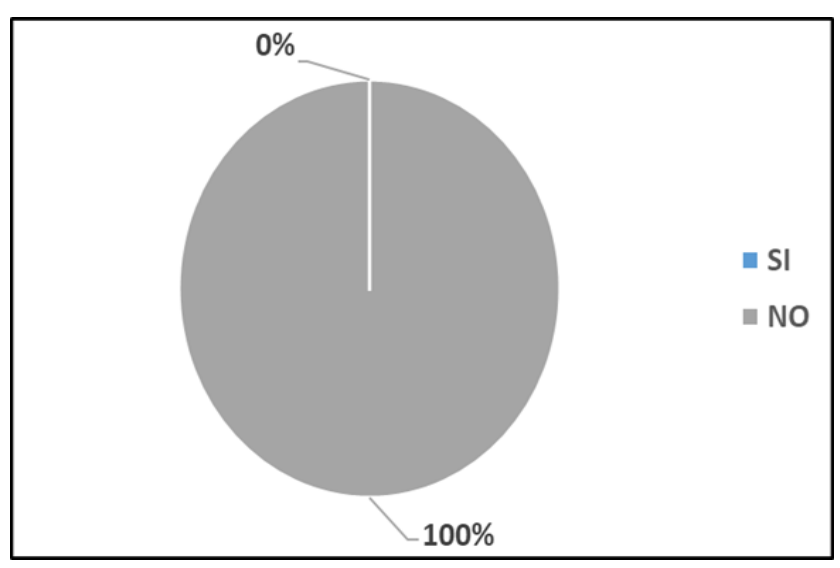

\section{Gráfica 4.}

Fuente: Resultado de la encuesta. Elaboración Propia (2020).

Para los encuestados, no se podría aplicar la sana crítica para emitir una sentencia si se verifica que la evidencia digital ha sido manipulada, constituyéndose este factor en elemento determinante del alto grado de errores al momento de determinar la responsabilidad de la persona procesada. 
5. ¿Cuál es su grado de conocimiento respecto a las características de las herramientas tecnológicas sobre informática forense?

\section{Tabla 5.}

\begin{tabular}{|l|c|c|}
\hline OPCIONES & NÚMERO & PORCENTAJE \\
\hline ALTO & 0 & $0 \%$ \\
\hline MEDIO & 15 & $16 \%$ \\
\hline BAJO & 30 & $31 \%$ \\
\hline NINGUNO & 51 & $53 \%$ \\
\hline TOTAL & 96 & $100 \%$ \\
\hline
\end{tabular}

Fuente: Resultado de la encuesta. Elaboración Propia (2020).

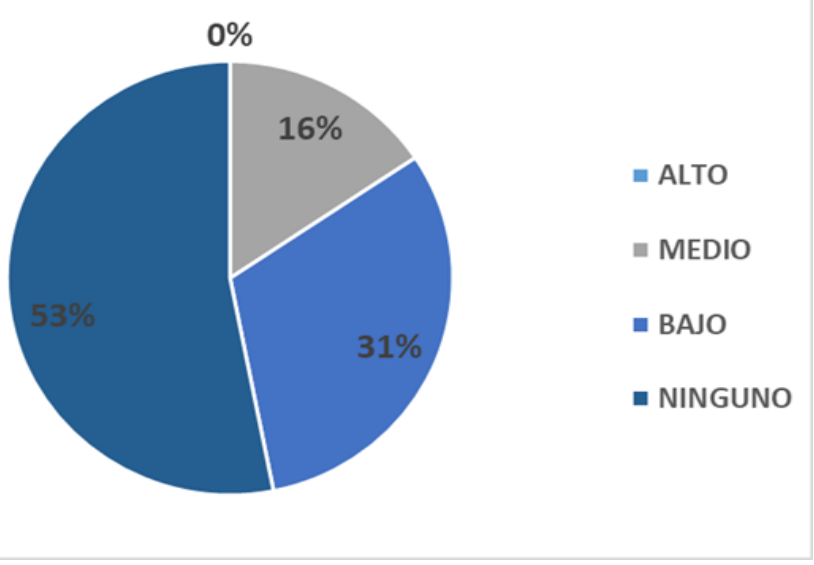

\section{Gráfico 5.}

Fuente: Resultado de la encuesta. Elaboración Propia (2020).

La necesidad de lineamientos claros que procuren mejorar los conocimientos respecto al uso de las herramientas tecnológicas sobre informática forense, es una realidad considera muy en cuenta por los involucrados en el trabajo de control y preservación de la evidencia digital. 
6. ¿Cuál es su nivel de conocimientos sobre los protocolos y estándares establecidos para la correcta cadena de custodia de la evidencia digital?

Tabla 6.

\begin{tabular}{|l|c|c|}
\hline OPCIONES & NÚMERO & PORCENTAJE \\
\hline ALTO & 0 & $0 \%$ \\
\hline MEDIO & 15 & $19 \%$ \\
\hline BAJO & 25 & $31 \%$ \\
\hline NINGUNO & 40 & $50 \%$ \\
\hline TOTAL & 80 & $100 \%$ \\
\hline
\end{tabular}

Fuente: Resultado de la encuesta. Elaboración Propia (2020).

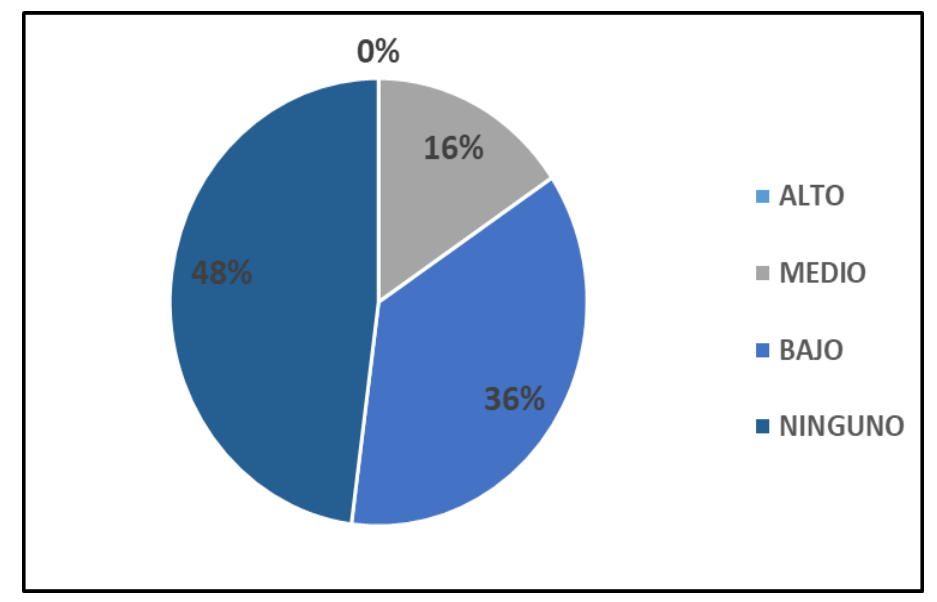

\section{Gráfica 6.}

Fuente: Resultado de la encuesta. Elaboración Propia (2020).

Los encuestados, en su gran mayoría, consideran muy deficiente el nivel de conocimientos sobre los protocolos y estándares establecidos para la correcta cadena de custodia de la evidencia digital, pudiendo mejorar dichos controles. 


\section{DISCUSIÓN}

Con el antecedente expuesto se determinan a continuación las inconsistencias que existen en el proceso de cadena de custodia y su relevancia en las causas relacionadas con delitos informáticos:

\section{Desconocimiento del procedimiento y protocolos}

El hecho de no tener la noción suficiente en la aplicación de protocolos de cadena de custodia de la evidencia digital deriva en cometer errores durante alguna etapa dentro del procedimiento a seguir, generando omisiones en la diligencia, en la definición y validación del formato del informe con los datos recolectados. Por ello, el cumplir con un adecuado procedimiento y contar con personal calificado, asegura la credibilidad, autenticidad e integridad de la investigación realizada (Proaño-Escalante, R. \& Gavilanes-Molina, A., 2018)

\section{Falta de herramientas adecuadas - Software}

Las herramientas de software con las que se cuenta según el "Manual de Manejo de Evidencias Digitales y Entornos Informáticos" no son las más adecuadas, ya que no cumplen con los requerimientos de innovación que existen en la actualidad ya que los mismos no aseguran que la evidencia adquirida pueda mantener su valor probatorio.

\section{Falta de herramientas adecuadas - Hardware}

La propuesta va encaminada a la necesidad de contar con la infraestructura de equipos informáticos que el proceso de cadena de custodia en la actualidad requiere, ya que en algunos casos ocurrirían inconsistencias con el uso de equipos obsoletos y sin la suficiente capacidad de almacenamiento dependiendo para lo que sea requerido. Discos duro y memorias externas, identificadores de código QR, rotuladores de evidencia. 


\section{Manejo inadecuado}

Se ha comprobado que las omisiones en la manipulación, almacenamiento, control y distribución de la evidencia digital en las diferentes etapas, se puede convertir en un factor concluyente para el desgaste del valor probatorio de la evidencia digital. Es fundamental considerar que el propósito de los protocolos de la cadena de custodia es procurar la probidad de la prueba o evidencia digital desde su conservación hasta que ésta sea trasladada a instancias siguientes dentro del proceso considerando anular la contaminación de la prueba para que esta atesore su valor dentro de la investigación.

Los protocolos de cadena de custodia tienen la tarea primordial de preservar las pruebas encontradas como resultado de la investigación realizada en una escena de crimen, considerando las características de veracidad, confiabilidad e idoneidad demostrables por cualquier perito especializado que tenga como tarea la realización de las respectivas pruebas con el uso de herramientas especializadas.

\section{CONCLUSIONES}

Como resultado el trabajo realizado nos permite llegar a la conclusión de que el personal especializado de la Policía Nacional no tiene conocimientos actualizados en el uso de la tecnológica para custodiar las evidencias digitales.

Se evidencia que la percepción mayoritaria es que la manipulación sin protocolos de seguridad de los indicios identificados, deriva en la modificación de la prueba provocando una inminente contaminación de la misma.

La capacitación sobre los nuevos procedimientos de custodia de evidencia digital es una necesidad urgente en el cantón Babahoyo para lograr un efectivo control y aplicación de los procedimientos en la preservación de la evidencia digital.

No se podría aplicar la sana critica para emitir una sentencia si se verifica que la evidencia digital ha sido manipulada, constituyéndose este factor en elemento determinante del alto grado de errores al momento de determinar la responsabilidad de la persona procesada. 
La necesidad de lineamientos claros que procuren mejorar los conocimientos respecto al uso de las herramientas tecnológicas sobre informática forense es una realidad considerada por los involucrados en el trabajo de control y preservación de la evidencia digital, considerando que existe un deficiente nivel de conocimientos sobre los protocolos y estándares establecidos para la correcta cadena de custodia de la evidencia digital, pudiendo mejorar dichos controles.

Desde la obtención hasta su procesamiento, se ha podido evidenciar la existencia de falencias en este proceso lo cual puede llevar a que las pruebas sean destruidas, manipuladas indebidamente o que pierdan su valor probatorio para los procesos judiciales.

\section{FINANCIAMIENTO}

No monetario.

\section{AGRADECIMIENTO}

A la Universidad Regional Autónoma de Los Andes; por motivar el desarrollo de la Investigación.

\section{REFERENCIAS CONSULTADAS}

Acurio del Pino, S. (2015). Manual de Manejo de Evidencias Digitales y Entornos Informáticos. Sistema especializado integral de investigación, medicina legal y ciencias forenses. [Manual of Digital Evidence Management and Computer Environments. Comprehensive specialized system of research, legal medicine and forensic sciences]. Fiscalia General del Estado. Quito - Ecuador. Recuperdo de https://n9.cl//4agl

Asamblea Nacional Constituyente de la República del Ecuador, (2008). Constitución de la República del Ecuador. [Constitution of the Republic of Ecuador]. Montecristi. Registro Oficial 449 de 20-oct-2008. Recuperado de https://n9.cl/sia

Bernal-Torres, C. A. (2006). Metodología de la investigación. [Research methodology] Recuperado de https://n9.cl/8txy. 
Calderon-Arias, E. (2014). Un estudio comparado en Latinoamérica sobre la cadena de custodia de las videncias en el proceso penal. [ A comparative study in Latin America on the chain of custody of vines in the grief process] FACULTAD DE DERECHO Y CIENCIAS POLÍTICAS. 44(121),425-459 Recuperado de https://n9.cl/tkwdw

Coello-Hernández, B. (2017). El sistema de cadena de custodia en el ecuador y sus efectos dentro del proceso penal. [The chain of custody system at the equator and its effects within criminal proceedings]. Universidad Autónoma de los Andes. Riobamba - Ecuador. Recuperado de: https://n9.cl/zkn3

Escobar-De León, L. (2017) Manejo de la cadena de custodia en la recolección de evidencia digital. [Chain of custody management in digital evidence collection] Tesis. Para optar al grado de licenciado en investigación criminal y forense. Universidad Rafael Landívar. Recuperado de: https://n9.cl/vama

Palella Stracuzzi, S. \& Martins Pestana, F. (2012). Metodología de la investigación cuantitativa. [Quantitative research methodology]. Fondo editorial de la Universidad Pedagógica Libertador. Caracas, Venezuela.

Proaño-Escalante, R. \& Gavilanes-Molina, A (2018) Estrategia para responder a incidentes de inseguridad informática ambientado en la legalidad ecuatoriana (Strategy for responding to computer incidents of insecurity set in Ecuadorian law). Enfoque UTE. 9(1),90-101. Recuperado de: https://n9.cl/lyy0

Sampaoli, J. (2018) Peritaje informático: marco teórico-práctico. [Computer expertise: theoretical-practical framework]. Tesis de Licenciatura en Sistemas y Computación. Universidad Católica Argentina. Facultad de Química e Ingeniería "Fray Rogelio Bacon". Recuperado de https://n9.cl/uwb3n

Serrano-Hernández, A., Serrano-Gómez, A. \& Serrano-Gómez, A. (2017) Medicina Legal e Informática: una aplicación para la gestión de la información [Legal Medicine and Informatics: an application for information management] Revista de Ciencias Médicas de Pinar del Río. 21(6), 820-827. Recuperado de: https://n9.cl/a265b 